\section{Toxic Metabolic Encephalo- pathy presenting as Bilateral Symmetrical Basal Ganglia Lesion}

Sir,

A 50-year male was brought to the hospital after being found unresponsive at home. Naloxone was administered by Emergency Medical Services (EMS) with no change in mental status. He was intubated to protect the airway and was normotensive at the time of presentation. Urine toxicology screen was positive for methamphetamine. CT scan at the time of admission was unremarkable for acute abnormality. Past medical history was only significant for polysubstance abuse, including cocaine and methamphetamine and alcohol withdrawal seizures. Electroencephalogram (EEG) ruled out epileptic focus. His mental status showed no improvement in two days and the MRI brain was ordered, which showed extensive bilateral symmetrical infarcts within basal ganglia, primarily within the caudate head and body, putamen, and internal capsules and cerebellum along with areas of hemorrhagic conversion (Figures 1 and 2). The trans-esophageal echocardiogram (TEE) and cerebrospinal fluid (CSF) analysis was unremarkable. Given his history of polysubstance abuse and symmetrical pattern, a diagnosis of toxic metabolic encephalopathy was made. The patient did not make meaningful neurological recovery and died after making comfort-care by the family.

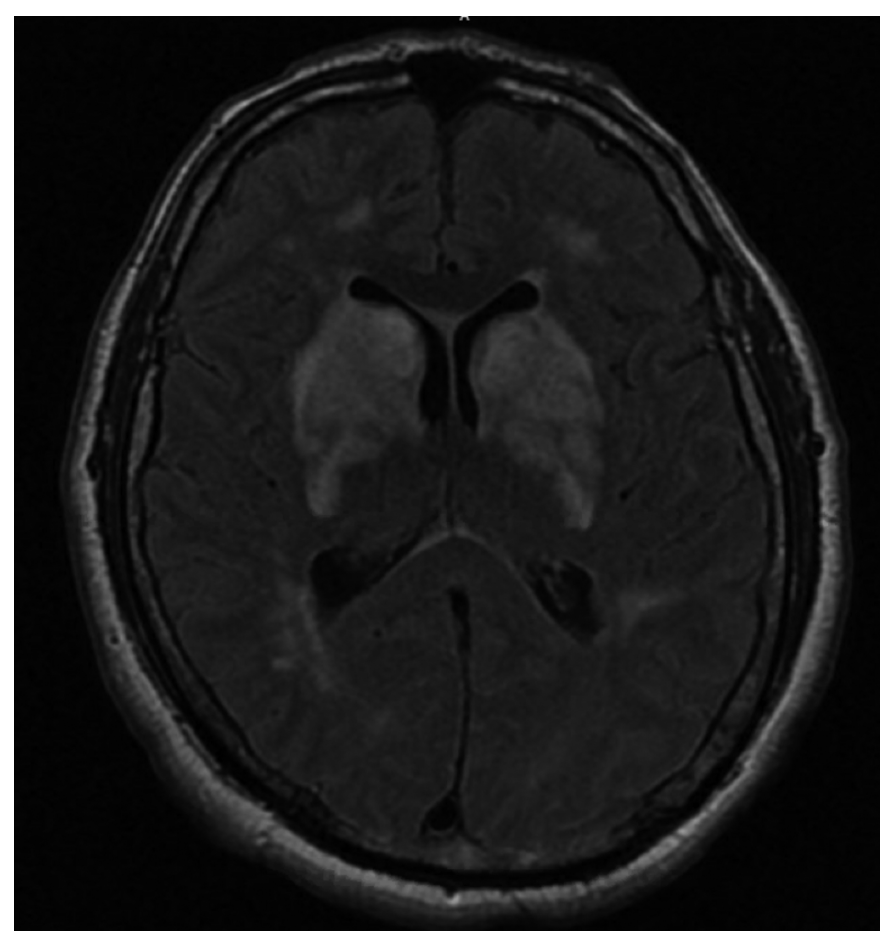

Figure 1: MRI scan of the brain in axial view at thalamic section with T2 FLAIR sequence showing infarcts in the bilateral basal ganglia.

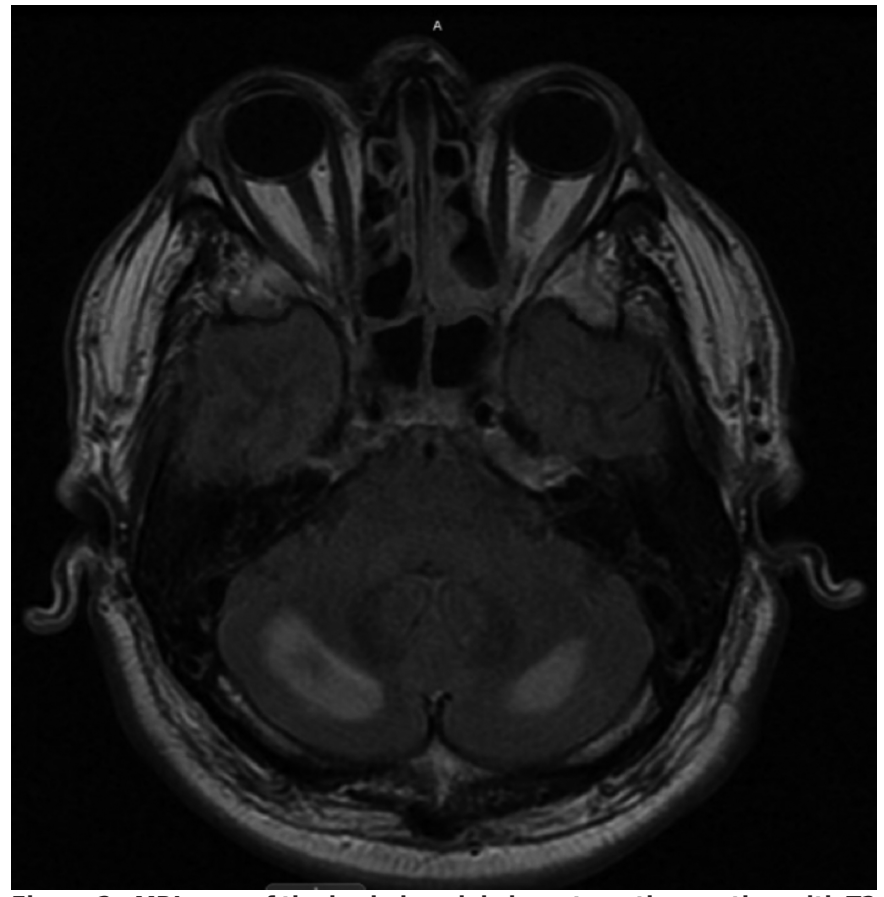

Figure 2: MRI scan of the brain in axial view at pontine section with T2 FLAIR sequence showing bilateral symmetrical infarcts in the cerebellum.

Acute unilateral infarctions, limited to the lenticular nuclei, are quite common and are often an incidental finding on imaging scans in patients with microangiopathy. Such lesions rarely cause clinical symptoms. Bilateral symmetrical acute lesions in the areas of the brain supplied by the perforating lenticulostriate arteries (basal ganglia, internal capsule, thalamus which are part of deep grey matter nuclei $\{D G M N\}$ ) have rarely been reported and favour metabolic etiology rather than vascular one and consist of wide differentials including hepatic, anoxia, hypoperfusion, hypoglycemia, infections or toxicity from carbon monoxide, methanol and recreational drugs among others. ${ }^{1}$ These lesions may occur acutely or may be part of a chronic process. ${ }^{1}$ Methamphetamine and cocaine are potent sympathomimetics that may lead to vascular events including myocardial infarction and stroke with the incidence of hemorrhagic stroke greater than ischemic stroke. Methamphetamine can also cause delayed ischemic stroke. ${ }^{2}$ The cause of brain damage may be multifactorial, related to associated vasculitis, direct toxic effects, accelerated atherosclerosis, and hypertensive crisis. ${ }^{3}$ In the background history of polysubstance abuse in this patient, bilateral basal ganglia infarctions could be explained by the illicit drugs. Drug testing should be considered in young people coming with stroke symptoms with no other identifiable etiology. The use of pertinent history, clinical course, selective laboratory investigation, and appreciation of neuroimaging abnormalities should be combined to define the etiology and prognosis.

\section{CONFLICT OF INTEREST:}

The authors declared no conflict of interest. 


\section{AUTHORS' CONTRIBUTION:}

SY: Conception, identifying the case, editing, obtaining figures.

$\mathrm{KM}$ : Writing discussion part and proofreading.

\section{REFERENCES}

1. Finelli PF, DiMario FJ. Diagnostic approach in patients with symmetric imaging lesions of the deep gray nuclei. Neurologist 2003; 9(5):250-61. doi: 10.1097/01.nrl. 0000087718.55597.6a.

2. Ohta K, Mori M, Yoritaka A, Okamoto K, Kishida S. Delayed ischemic stroke associated with methamphetamine use. J Emerg Med 2005; 28(2):165-7. doi: 10.1016/j.jemermed. 2004.06.015.

3. Ho EL, Josephson SA, Lee HS, Smith WS. Cerebrovascular complications of methamphetamine abuse. Neurocrit Care 2009; 10(3):295-305. doi: 10.1007/s12028-008-9177-5.
Saddam Yasin ${ }^{1}$ and Kanwal Mehmood ${ }^{2}$

${ }^{1}$ Department of Internal Medicine, Carle Foundation Hospital, Urbana, United States of America

${ }^{2}$ Department of General Medicine, Shalimar Medical and Dental College, Lahore, Pakistan

Correspondence to: Dr. Saddam Yasin, Department of Internal Medicine, Carle Foundation Hospital, Urbana, United States of America

E-mail: saddamyasinmd@gmail.com

Received: August 07, 2020; Revised: November 27, 2020; Accepted: December 11, 2020

DOI: https://doi.org/10.29271/jcpsp.2021.10.1259 